\title{
Miniaturized data loggers for deep sea sediment temperature gradient measurements
}

\author{
Marion Pfender*, Heinrich Villinger \\ Fachbereich Geowissenschaften, Universität Bremen, P.O. Box 330440, D-28334 Bremen, Germany
}

Received 26 September 2001; accepted 8 March 2002

\begin{abstract}
A new probe for autonomous high precision temperature measurements in deep sea sediments has been developed by Fa. Antares and the University of Bremen and extensively tested during several expeditions. The miniaturized temperature data loggers (MTLs) were constructed to be extremely robust, small $(16 \mathrm{~cm}$ long) and easy to operate in waterdepths up to $6000 \mathrm{~m}$. The temperature range extends from -5 to $+60^{\circ} \mathrm{C}$ with an absolute accuracy of $\pm 0.1 \mathrm{~K}$. Higher absolute accuracy requires calibration with a high precision thermometer. The resolution of the $\mathrm{A} / \mathrm{D}$ converter is 16 bit which leads to a temperature resolution of $0.001 \mathrm{~K}$. The logging duration and sampling rate can be chosen independently. Up to $18 \mathrm{~h}$ of measurement are possible with the highest sample rate of $1 \mathrm{~s}$. Data are stored in the non-volatile memory which holds up to 64800 measurements. The probe is configured and data downloaded without opening the pressure case by using specialized software and interface hardware. The MTLs offer a wide variety of marine science applications wherever a high precision temperature measurement is of interest. Our main application is the investigation of temperature gradients in deep sea sediments where measurements of gradients over a large depth interval are required by fixing loggers on either different kinds of core barrels or on long lances. During four expeditions, we used the loggers to measure sediment temperature gradients in several geological settings. The design of the attachments to the different carriers was varied to optimize the results for different needs. These tests were highly successful and the data of high quality. Therefore the loggers can be recommended for regular use in marine applications. (C) 2002 Elsevier Science B.V. All rights reserved.
\end{abstract}

Keywords: temperature gradients; deep sea sediments; heat flow; temperature data logger

\section{Introduction}

The growing interest in global and geochemical energy fluxes in the oceans has led to a remarkable increase in marine geothermal studies. These are mainly focused on the sediment-covered areas

* Corresponding author.

E-mail addresses: pfender@geophys2.uni-bremen.de (M. Pfender), vill@uni-bremen.de (H. Villinger). of ridges and ridge flanks. Numerous studies of accretionary wedges also incorporate heat flow investigations, reflecting the need for additional constraints to model and understand the dewatering process of accreted sediments and gas hydrate formation. The extension of hydrocarbon exploration into deeper water in recent years triggered a growing demand in industry for heat flow measurements as an additional constraint in their evaluation of hydrocarbon occurrence.

This increased demand for marine heat flow 
data has helped to improve the existing conventional measurement technique in two ways: (1) the violin bow type heat probe instrument, as described for example by Hyndman et al. (1979), has evolved over a decade of intensive use from a prototype to a mature, mechanically robust instrument which now can be used in a routine way, (2) the progress in electronics has increased the true temperature resolution to $1 \mathrm{mK}$ and the increased data storage capacity has allowed more sensors. Both developments now permit multi-penetration deployments (measurements in a pogo style fashion) of up to $24 \mathrm{~h}$. A single heat flow determination consists of a temperature gradient measurement typically over $3-5-\mathrm{m}$ sub-seafloor probe penetration and an in situ measurement of thermal conductivity. Both results are then combined to determine the heat flow. Details can be found in Hyndman et al. (1979) or Kaul et al. (2000). An additional improvement comes from dynamic positioning of research vessels using the Global Positioning System (GPS or DGPS) which helps enormously in station keeping during a measurement and positioning of the probe for very detailed heat flow investigations.

However, the use of a conventional heat probe has several drawbacks which may prevent scientists from using it:

- currently used heat probes are highly sophisticated and expensive instruments and require a high degree of technical maintenance and welltrained personnel and therefore only a few groups worldwide support these instruments;

- most conventional heat probes have a subseafloor penetration depth of only 3-4 $\mathrm{m}$ due to mechanical limitations; this creates severe problems when working in areas where bottom water temperatures vary with time and create disturbances of the shallow, sub-seafloor temperature field;

- conventional heat flow surveys are time-consuming and therefore expensive.

Temperature sensors attached to the outside walls of a sediment core barrel may provide a solution to these drawbacks. Because sediment core barrels are typically 5-20 m long, temperature gradients can be measured over a substantially larger depth interval when taking a core.
In order to calculate heat flow, thermal conductivity has to be measured for example with needle probes (Von Herzen and Maxell, 1959; Lister, 1979) on recovered sediment cores. An advantage of this method is that at each coring station one could measure a temperature gradient and potentially get a heat flow value without the need of additional ship time. In the case of hydrothermal studies also incorporating pore water chemistry profiling, the temperature gradients provide constraints for deriving advection rates through the sediments.

However, this approach of measuring temperature gradients using outrigger sensors is not new. Typically, when this method was used in the past, each individual sensor (outrigger) was connected to one central data logging unit (Langseth, 1965; Gerard et al., 1962), housed in the weight stand of the corer. Problems with leaks at high pressures and frequent damage of the fragile outriggers and cables during launch and recovery of the coring system discouraged scientists to use such a setup; the last results with such a classical outrigger system were published by Lister et al. (1990). Fisher et al. (1996) used autonomous outrigger probes to measure thermal gradients on piston and gravity cores, but although the instruments performed well at sea, the electronics deteriorated over time.

In 1999 we started an instrument development project together with a Bremen-based company, Antares, which specializes in downhole logging equipment. The basic design philosophy was to develop a miniaturized, robust and self-contained single channel temperature data logger, which can be easily attached to the external wall of a core barrel and can also be used for other temperature monitoring tasks in a deep sea environment. The first prototype instruments were tested on a research cruise aboard the German R/V 'Sonne' at the end of 1999/2000 (Villinger et al., 2000). Small design problems in hard- and software were eliminated after these extensive tests and the loggers were used again during cruises SO146 (Bialas et al., 2000) and SO149 (Spiess et al., 2001) in 2000. The first measurements on a routine basis were done during the cruise EW0104 on board the American R/V 'Maurice Ewing' in 2001 (Fisher et al., 2001a,b). In the following we will describe 


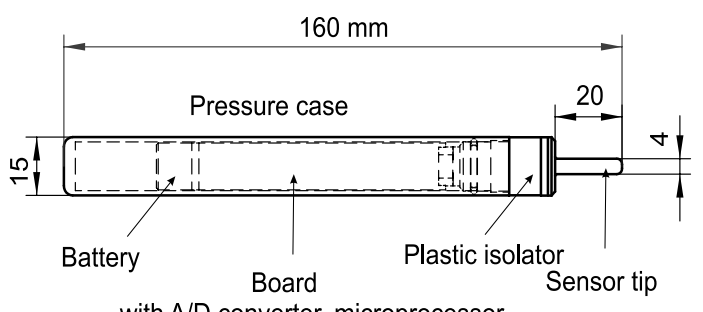

with $A / D$ converter, microprocessor, internal clock and memory

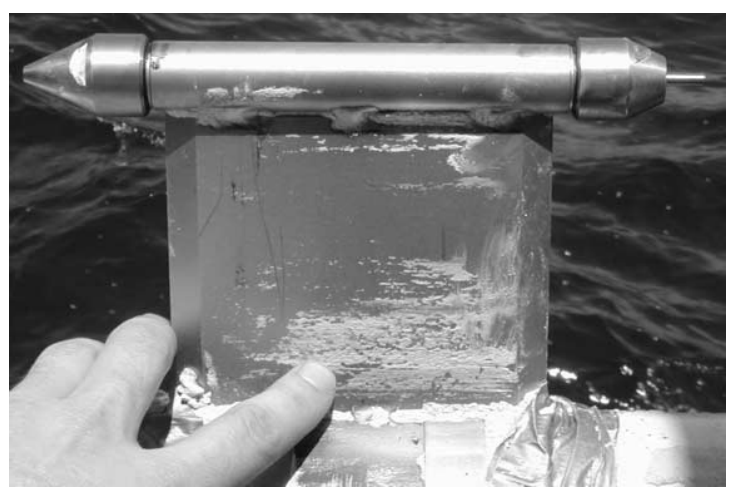

Fig. 1. Upper: construction sketch of a logger. Lower: logger protected in a fin-like attachment mounted on a core barrel.

the instrument in detail, show its use for measuring temperature gradients in sediments and discuss its use for other types of temperature monitoring in a deep sea environment.

\section{Detailed description of logger features}

\subsection{Construction}

The miniaturized temperature data logger (MTL) consists of a 140-mm-long cylindrical body with an outside diameter of $15 \mathrm{~mm}$, housing the electronics, and a thin-walled tip (20 $\mathrm{mm}$ long with an outer diameter (o.d.) of $4 \mathrm{~mm}$ ) containing the temperature sensor. The design of a temperature sensor in a thin-walled tip satisfies the need for a fast response sensor. The pressure housing consists of high-strength corrosion resistant steel and withstands a pressure equivalent of $6000 \mathrm{~m}$ waterdepth. The logger is shown in Fig. 1.

Programming of the logger and downloading the data are performed without opening the pres- sure case. A readout unit contacts the logger's tip and end cap with a voltage delivered by an RS232 interface from a PC and a high-strength plastic isolator between tip and main body maintains the voltage so that the communication unit works as a two point connection for data transfer. Therefore, the logger has to be opened only to replace the battery which is located beneath the end cap.

\subsection{Electronics}

The electronics of the logger consist of a microprocessor, a 16-bit A/D converter, a real-time clock and non-volatile memory. The complete system is powered by a standard small-sized 3-V lithium battery. We use a thermistor (interchangeability of $0.1 \mathrm{~K}$ ) as a sensing element in order to achieve a resolution of $1 \mathrm{mK}$ at typical deep sea temperatures of $2^{\circ} \mathrm{C}$. The thermistor is part of a bridge whose offset voltage provides the signal that is digitized and stored. In addition to temperature, the time of the first reading, the total number of measurements made with an individual battery and the battery voltage at the beginning and end of logging are stored as well. The memory can hold up to 64800 measurements, which allows up to $18 \mathrm{~h}$ of logging at a 1-s interval. The sample interval can be varied from $1 \mathrm{~s}$ to $255 \mathrm{~min}$, so the longest theoretical recording time is around $30 \mathrm{yr}$. A loss of battery voltage beneath the required $2.7 \mathrm{~V}$ neither produces misleading temperature readings nor influences the reliability of the measurement, but creates a constant error value of $-5^{\circ} \mathrm{C}$ before the record stops. Data recorded until then are stored in the non-volatile memory.

Programming and data transfer between logger and PC are performed via a serial link (RS232) by placing the logger in a readout unit and using the WINTEMP software. The GUI software allows the user to set up logging parameters such as start and end of logging period and sample interval, and to set the logger clock. Once the data are downloaded the software automatically converts the raw logger readings (bits) into thermistor resistances and equivalent temperatures. This conversion is based on individual calibration files for each logger, supplied by the manufacturer. 


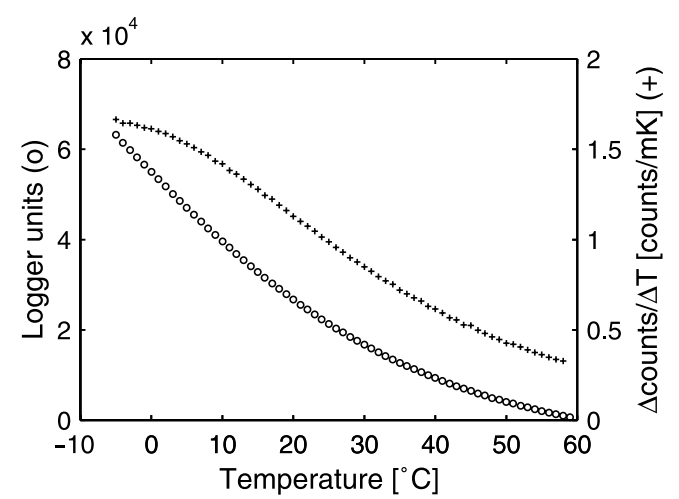

Fig. 2. Logger's characteristic (circles) and sensitivity (crosses). The characteristic is the A/D converter output in dependence on the environmental temperature and the sensitivity is the derivative $\partial$ counts/ $\partial T$. Sensitivity is highest at deep sea temperatures.

Data can be either stored in an internal binary format or exported as a column-oriented ASCII data file consisting of header information and the data itself. Thus, the file can easily be loaded in any spreadsheet program for further processing.

\subsection{Temperature measurement characteristics and calibration}

The temperature measurement range extends from -5 to $+60^{\circ} \mathrm{C}$ with a maximum resolution of $1 \mathrm{mK}$. Fig. 2 illustrates both the logger's raw data output (circles) and its sensitivity (crosses) versus temperature. These data were produced by replacing the thermistor with a highly stable decade resistance box to simulate the thermistor characteristic. The raw data output varies from 60000 counts at $-5^{\circ} \mathrm{C}$ to nearly 0 at $+60^{\circ} \mathrm{C}$. The derivative dcounts/ $\partial T$ - the sensitivity - is inversely related to temperature and lies well above 1.5 counts $/ \mathrm{mK}$ for most marine applications, i.e. for temperatures between 0 and $5^{\circ} \mathrm{C}$.

To characterize the response time of a sensor to an abrupt change in temperature, the thermal time constant is generally used. It is defined as the time required for a thermistor to change its body temperature by $63.2 \%$ of a specific temperature span. To estimate the thermal time constant, we rapidly immersed the logger from hot to cold water. The sensors's response in normalized temperatures is shown in Fig. 3. In this case, the temperature change by $63 \%$ is reached after $2 \mathrm{~s}$ which is the thermal time constant of the system.

A first order temperature calibration is performed by default using the communication software WINTEMP. It is based on the thermistor spectra and the Steinhart-Hart relation (Steinhart and Hart, 1968; Bennett, 1972) and uses logger specific calibration coefficients included in the software. The absolute accuracy of this standard calibration is $0.1 \mathrm{~K}$ which corresponds to the interchangeability of the thermistors.

In practice, we increase the absolute accuracy of the thermistors through a more precise calibration by equating the logger's output to a high precision thermometer in a well-stirred water bath whose temperature is increased in predefined steps. The results are presented in Fig. 4: the upper part shows the reference temperature as a function of the logger readings and a polynomial fifth degree fit (solid line) of raw data to reference temperature. The lower section shows the residuals between the fitted logger output and reference temperature. This calibration yields an improvement of the absolute accuracy to several $\mathrm{mK}$ which is sufficient for measurement of marine sediment temperature gradients.

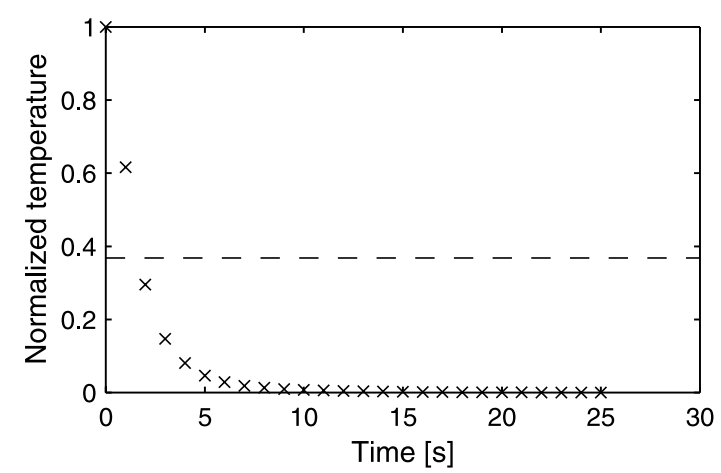

Fig. 3. Response of a sensor to a rapid change in temperature. The dashed line indicates the temperature decreased by $63.2 \%$ of the given temperature span following the definition of the thermal time constant. The resulting time constant is $2 \mathrm{~s}$. 

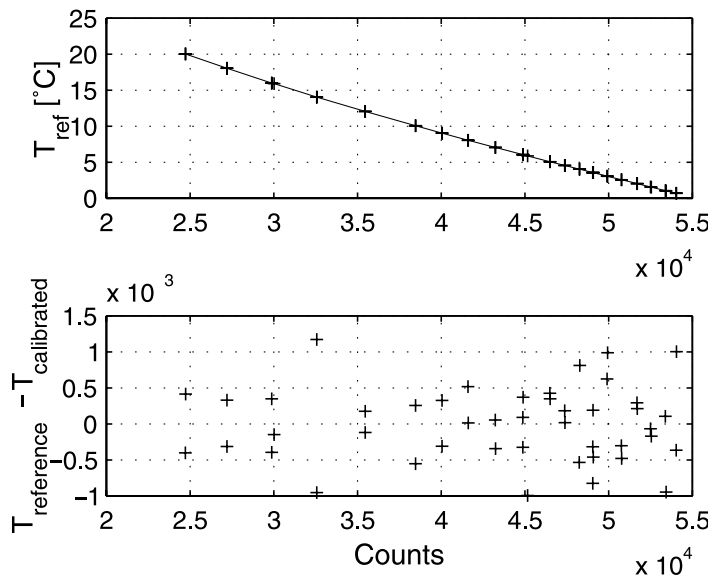

Fig. 4. Upper: reference temperature versus logger's raw data output (crosses) and fifth degree polynomial fit (line). Lower: residuals between reference temperature and logger's temperature output after calibration with fifth degree calibration polynomial.

\section{Temperature gradient measurements using MTLS}

\subsection{Introduction}

In this section, we describe the practical use of the MTLs to measure temperature gradients in marine sediments.

Two different ways of gradient measurements have been tested on four expeditions:

- The MTLs are attached to a 6-m steel lance which has the same o.d. - $63.5 \mathrm{~mm}$ - as the conventional heat probe's strength member. The advantage of this deployment is that temperature gradients over a larger depth interval compared to the conventional probe can be measured. The lance has not to be recovered between the single penetrations, but can be used in the time-saving so-called pogo style. With this method, fast overview measurements in large areas as well as detailed investigations with a high spatial resolution are possible. On the other hand, information concerning the thermal conductivity cannot be obtained from this investigation. To calculate heat flow, a mean value of known conductivity from the investigation area has to be considered.

- The MTLs are attached to gravity and piston core barrels which cannot be used in pogo style but have the advantage that a temperature gradient can be obtained over a larger depth interval with each deployment of the corer without additional ship time. Moreover, the thermal conductivity can be measured on the recovered core material for example with the needle probe method.

A critical part is the attachment of the loggers to their carrier which has to fulfill two aspects: on the one hand it has to be robust and protect the logger against damage, on the other hand it must guarantee a small thermal time constant of the whole system. Our experiences have shown that a fin-like attachment as in Fig. 1 is the best solution for this trade-off. The fin holds the logger $10 \mathrm{~cm}$ away from the carrier; this distance corresponds to the distance of the sensor tube to the strength member of the conventional heat flow probe. The MTL itself is mounted in a cylindrical housing on top of the fin. Its 20-mm-long sensor tip protrudes $12 \mathrm{~mm}$ out of the outer housing which guarantees a fast temperature adaptation but provides enough protection against severe damage during penetration. The fins are mounted on their carrier either with screws or stainless steel bandings at changing angles to allow the penetration of each sensor into undisturbed sediment. The exact positions of the fins relative to a fixed point, for example the edge of the weight stand, have to be thoroughly measured before and after each deployment.

The preparations for deployment on the ship are the same for the different carriers: the loggers are programmed with start time and sample rate and, as a prerequisite to guarantee the comparability of the single records, their internal clocks synchronized. In order to achieve a high temporal resolution, we chose a sample rate of $1 \mathrm{~s}$ in all cases which allows a logging duration of $18 \mathrm{~h}$ which was enough even for the pogo style profiles we measured. All our loggers in use are calibrated so that the absolute accuracy is several $\mathrm{mK}$. In addition, the battery voltage was checked before each deployment. The instruments are mounted in their housings and the corer or lance lowered to the seafloor. After penetration, the loggers have to stay in the sediment long enough to allow the temperature sensors to adapt to ambient temper- 

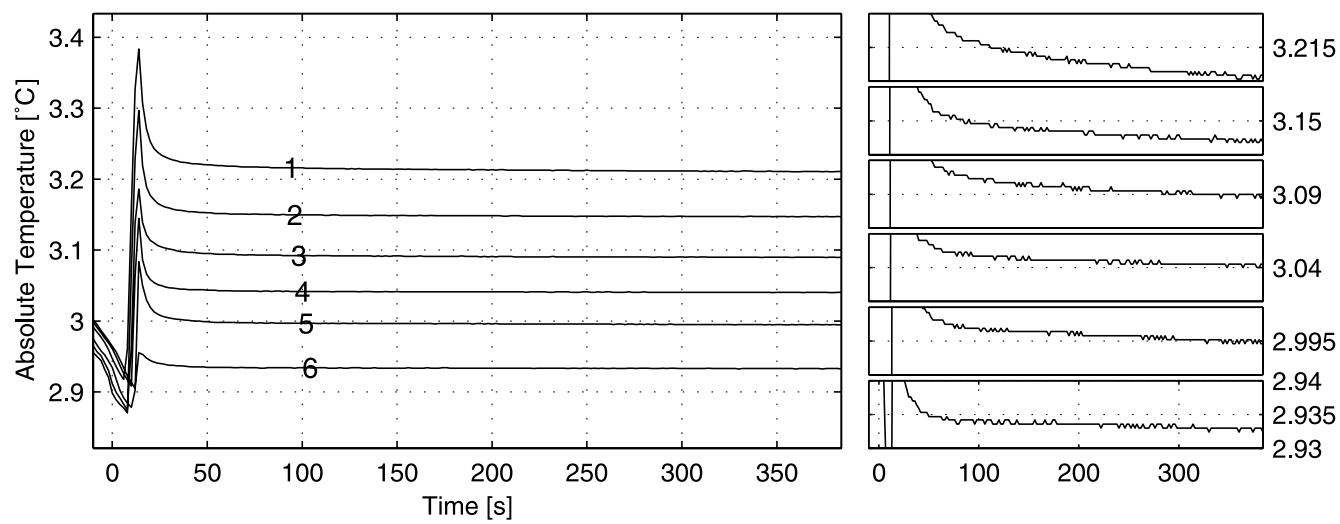

Fig. 5. Left: temperature records over $380 \mathrm{~s}$ of all six loggers during deployment of the 6-m lance. The records show the frictional heating with the following adaptation to sediment temperature. Right: enlargements of each logger's decay curve with the same time interval as the original plot on the left side and a temperature scale of $0.01^{\circ} \mathrm{C}$. The decay is free of disturbance and the scatter at the end of the record in the range of $\mathrm{mK}$ which is the digital noise.

ature, which is typically $3-5 \mathrm{~min}$. Then the carrier is pulled out of the sediment and - in case of the corer - recovered. If a lance is used instead of a corer, measurements can be performed on profiles in pogo style. Once the carrier is back on deck, the loggers are taken out of their housings and data are downloaded and processed.

\subsection{Pogo style measurements with a 6-m lance}

The first measurements were carried out in the area of the Juan de Fuca Ridge on cruise SO149 on board the German R/V 'Sonne'. For details concerning the goals of this cruise and locations of the lance measurements see the cruise report (Spiess et al., 2001). The lance was deployed on four profiles in different areas, the data we show here are from a test site on the Cascadia margin in a waterdepth of about $1300 \mathrm{~m}$. This profile with alltogether 12 penetrations was surveyed twice with both the lance and a conventional heat probe within $9 \mathrm{~h}$ in order to check the reliability of the results. The lance was equipped with six loggers in distances of $1 \mathrm{~m}$ arranged in a spiral design with angles of $60^{\circ}$. The lance was mounted in the conventional heat probe's weight stand.

An example of one penetration is shown in Fig. 5, where the graph on the left side contains all six temperature records versus logging time, and the graphs on the right side are enlargements for each single instrument to demonstrate the high data quality. In the left part of Fig. 5, prepenetration temperatures significantly decrease while the probe is lowered to the seafloor with a speed of about $1 \mathrm{~m} / \mathrm{s}$. This strong vertical temperature gradient in bottom water is a typical feature of shallow water measurements and stands in contrast to deep sea measurements where the vertical temperature gradient in bottom water is adiabatic and cannot be detected over the lance's length with the sensor's resolution. The bottom water temperature at the Cascadia margin location as measured prior to penetration is around $2.9^{\circ} \mathrm{C}$. The temperature rise - the frictional heating - is caused by penetration of the probe into the sediment. The following temperature decay and equilibration to ambient sediment temperature are recorded for the next $380 \mathrm{~s}$. The complete record is shown with an expanded and uniform temperature scale of $0.01 \mathrm{~K}$ on the right side of Fig. 5. The decays free of disturbances show that reliable data have been recorded over the complete time the loggers were resting in the sediments. The scatter in the records (see Fig. 5 right) is the digital noise in the range of $\mathrm{mK}$.

From the temperature decays, the temperature gradient can be calculated with different methods:

- Gradient calculation based on extrapolated undisturbed sediment temperatures.

This calculation is based on the long term ap- 
proximation (asymptotic solution) of the thermal decay curve of an infinitely long, infinitely conductive line source (Blackwell, 1954; Hyndman et al., 1979):

$T(t)=\frac{Q}{4 \pi k t}+T_{\mathrm{a}}$

with $Q$ amount of heat $(\mathrm{J} / \mathrm{m})$ produced by penetration, $k$ thermal conductivity $(\mathrm{W} / \mathrm{mK})$ and $T_{\mathrm{a}}$ ambient temperature (K).

Plotting temperatures over inverse time yield one curve for each single thermistor which becomes linear for large times. A linear regression of $T$ versus $1 / t$ in this time window gives $T_{\mathrm{a}}$ at $(1 / t)=0$, i.e. infinite time. Normally a time window of $100 \mathrm{~s}$ is used at the end of the data set. Shifting this window in time will result in a timedependent gradient as explained below.

- Gradient calculation directly from temperature readings.

Another possibility to quickly obtain results is based on the assumption that a time-dependent gradient, calculated from individual simultaneous temperature readings of the different sensors, asymptotically approaches the true gradient. This requires the precise synchronization of the MTLs before deployment.

Before calculating gradients for any penetration, it is necessary to investigate the influence of the penetration angle on the penetration depth. On the cruise SO149, a tilt sensor was not yet available for the lance, but is installed in the conventional heat probe. The mean tilt of all conventional heat probe penetrations during cruise SO149 was $1.5^{\circ}$ with a maximum value of $10^{\circ}$. As the gradient varies with the cosine of the tilt angle, all tilt values below $10^{\circ}$ result in a tilt-related uncertainty of less then $2 \%$. Therefore we neglect this effect and assume a vertical penetration of the lance. In the meantime MTLs equipped with tilt sensors are routinely mounted during deployments (see 3.3. Gradient measurements with a gravity corer).

A comparison of gradients derived with these two methods is illustrated in Fig. 6. The upper part of Fig. 6 shows the temperature decays, the lower figure illustrates the changing gradients with
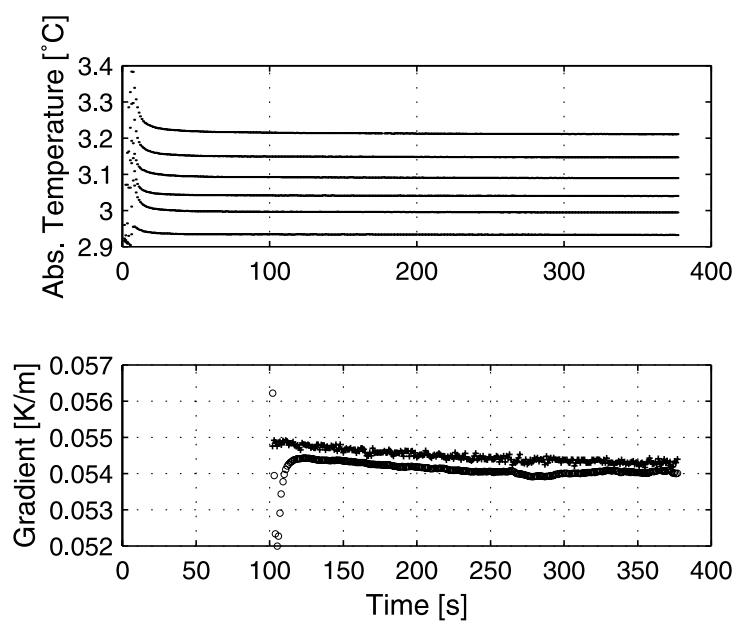

Fig. 6. Upper: temperature records versus logging time for all loggers like in Fig. 5. Lower: time-dependent gradients derived with the direct method (+) and extrapolation (o) to infinite times. The methods are described in the text. The result in the gradients does not vary more than $2 \%$ with recording time.

time, obtained with the two methods described above. To calculate the 'extrapolated' gradients over time (illustrated by circles), we used a moving time window of $100 \mathrm{~s}$ width and calculated the linear fit of temperature versus inverse time to obtain $T_{\mathrm{a}}$ for each sensor. The resulting time-dependent temperature gradient is associated with the time at the end of the time window. The 'directly' calculated gradient (illustrated by stars) from a linear regression of temperature readings versus depths is also calculated at the end of the above mentioned time window.

Both gradient curves in Fig. 6 show a decreasing gradient with increasing time which becomes stable after $300 \mathrm{~s}$. The changes of the calculated gradient with recording time do not exceed $1 \%$ in each case, but it becomes clear that times smaller than 120-150 s should not be taken into account for the extrapolation method because here the decay is mainly influenced by the lance geometry and not the surrounding sediment. Additionally, the long term approximation is not meant to work for small decay times. With increasing record length, the difference between the two gradients becomes smaller and is less than $1 \%$ at the final time window used. 


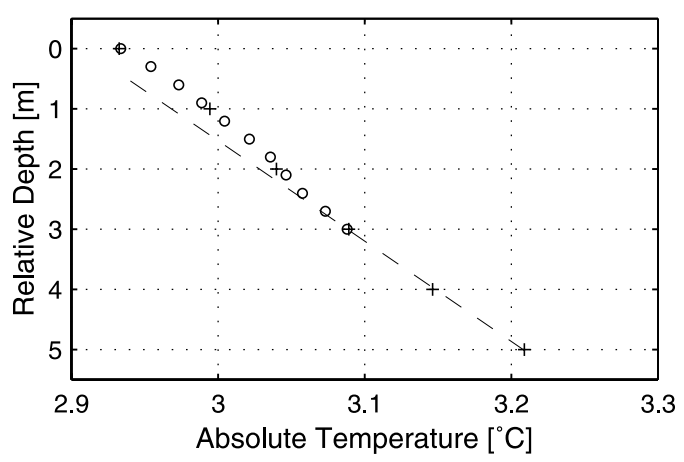

Fig. 7. Sediment temperatures obtained with heat flow probe (o) and temperature loggers mounted on a 6-m lance (+). The measurements have been performed at almost identical locations, the determined temperatures compare well. The gradient is disturbed in the upper meters and becomes free of disturbance in depths up to $5 \mathrm{~m}$. The dashed line indicates the undisturbed gradient.

The influence of small scale temperature variations in the decay curves of each logger on the gradients calculated with the two methods can be shown around $260 \mathrm{~s}$ in Fig. 6: while the direct method reacts to such a disturbance with a small scale scatter, the extrapolation method results in a longer lasting variation in the gradients, as can be seen in the dip from 270 to 300 s. In general the extrapolation method results in a smoother time dependence of the gradient but gives more emphasis on small scale temperature variations in the decay curve of each single sensor. Despite this different behavior, the difference between directly calculating the gradient from the data or extrapolating the sediment temperature does not exceed $2 \%$, so the direct method gives a fast and reliable result to estimate the gradient.

Since this measurement has been performed on a profile of conventional heat flow measurements made during the same day, the temperature profiles at two locations $60 \mathrm{~m}$ apart from each other can be compared. The results are plotted together in Fig. 7. As the absolute depth below seafloor is not known, this paper will refer to depth relative to the uppermost sensor in all cases. The depth correction for tilt is neglected as explained above. The circles indicate the conventional heat flow measurement which reaches up to a depth of $3 \mathrm{~m}$ relative to the first sensor while the crosses mark the logger measurements which reach up to a relative depth of $5 \mathrm{~m}$ (positions of the loggers relative to the weight stand: $1,2,3,4,5$ and $6 \mathrm{~m}$ ). The sediment temperatures in different depths compare very well to each other. The non-linear gradient in the upper meters caused by bottom water temperature changes is measured by both instruments. A comparison of the mean calculated gradients in this depths yields $0.0506 \mathrm{~K} / \mathrm{m}$ for the conventional probe and $0.0519 \mathrm{~K} / \mathrm{m}$ for the lance, which is a good agreement with a $3 \%$ deviation. The lance with the deeper penetration also measures the gradient in larger depths where it becomes linear and free of disturbances.

On cruise SO149 we successfully used the 6-m lance 28 times with six loggers mounted on it. The fin-like attachments were fixed on the lance with small screws which held the whole system in place during all measurements without any movement. No instrument was damaged or lost during these deployments. The data were of a high quality and could easily be processed. The 6-m lance is therefore very useful in areas where temperatures, measured with conventional short heat probes, are disturbed and it can be used on a routine basis. The heat flow can be calculated from the temperature gradient if the thermal conductivity is known by other methods.

\subsection{Gradient measurements with a gravity corer}

Experience with loggers mounted on gravity corers was gained on two different cruises: for the very first tests on the cruise SO145 to the East Pacific Rise and its flanks (for more details see cruise report: Villinger et al., 2000) we used sturdy attachments in order to guarantee a maximum of protection for the loggers. Unfortunately, these fixings produced severe temperature disturbances and therefore were not used again. On cruise EW0104 on board the American R/V 'Maurice Ewing' off Costa Rica (details can be found in the cruise report by Fisher et al., 2001a,b) we used the fin-like attachments as described in 3.1. Introduction in a routine way. Typically three outriggers were mounted on a 10 -feetlong, 2.5" o.d. diameter gravity core barrel with stainless steel bandings. Again the positions of the 

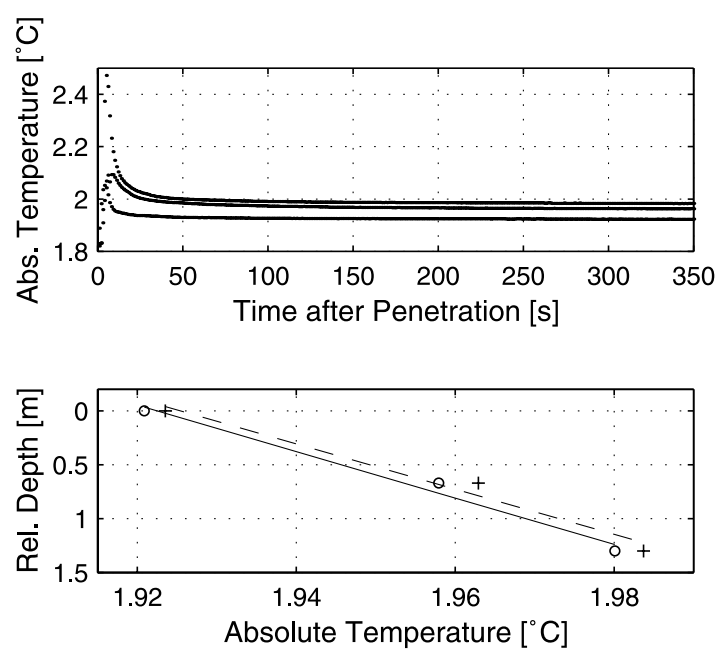

Fig. 8. Upper: temperature records over $350 \mathrm{~s}$ from coring station GC16. Lower: sediment temperatures derived with the direct method (+) and extrapolation (o). Both gradients are linear and have a difference of $3 \%$.

fins relative to a fixed point on the core barrel were observed before and after deployment.

Additionally, a tilt sensor built in a logger's pressure case was mounted in the weight stand. Its sensitivity allows only a rough estimation of the penetration angle in steps of $5^{\circ}$ and is more helpful to decide if a penetration was successful than to determine the exact penetration angle. Since the observed tilts in the whole investigation area were mostly well below $5^{\circ}$, we also ignore these angles and assume a vertical penetration for the discussed example.

An example of temperature records from instruments mounted $1.58,2.25$ and $2.88 \mathrm{~m}$ beneath the weight stand is shown in the upper part of Fig. 8. The temperature records of GC16 show the frictional heating and the undisturbed temperature decays which have been recorded for almost $6 \mathrm{~min}$. In the lower part of Fig. 8, the crosses indicate the sediment temperatures derived from the direct method and the slope of the dashed line is the mean gradient. The circles and full line illustrate the results of the extrapolation method. The gradient from the direct method is 0.048 $\mathrm{K} / \mathrm{m}$, from the $1 / t$ method $0.0465 \mathrm{~K} / \mathrm{m}$. The difference of each result to the mean gradient therefore is $2 \%$. As the thermal conductivity has not been determined on this core, a mean value of 0.74
$\mathrm{W} / \mathrm{mK}$ from the nearby conventional heat flow profile is assumed and the resulting heatflow is 35.5 and $34.4 \mathrm{~mW} / \mathrm{m}^{2}$, respectively.

Altogether, 10 coring stations were performed during the entire cruise EW0104 with gravity corers equipped with mostly three temperature data loggers. Mounting the attachments on the core barrel with the help of steel bandings was successful, but it generally requires more maintenance than the system with screws. Even if no visible motion took place, steel bandings which seem to have stretched during deployment have to be replaced for each new deployment. With this servicing, no sliding along the core barrel has been observed and no instrument was lost. Only two sensor tips were slightly bent during penetration and were replaced as a precaution in order to avoid further damage. Data obtained from the MTLs are of a high quality and not disturbed. The gradients could be calculated at all locations with one exception where the uppermost sensor did not penetrate. Since the core material has been used for pore water chemistry investigations, thermal conductivity could not be determined on the recovered core material. The conductivity had to be taken as a mean value from the closest conventional heat flow measurements, and the calculated heat flow fits well in the heat flow pattern of the investigation area. In four cases, the liner was empty although sediment marks on the outside walls, an inverted core catcher, a small tilt and frictional heating recorded by the loggers indicated a penetration of the core barrel. It is not clear if this loss of core material is influenced by the time the core barrel rested in the sediment, by the attachments themselves or only by coincidence.

\subsection{Gradient measurements with a piston corer}

On the cruise EW0104 we deployed the loggers on a 4 " diameter and 30-feet-long piston corer as well. A maximum of six loggers were mounted over $8.9 \mathrm{~m}$ length on the core barrel. Again we mounted the tilt sensor in the weight stand. The data we show in Fig. 9 are from a measurement with four loggers distributed over more than $5 \mathrm{~m}$ (depths relative to weight stand are 1.57, 2.83, 

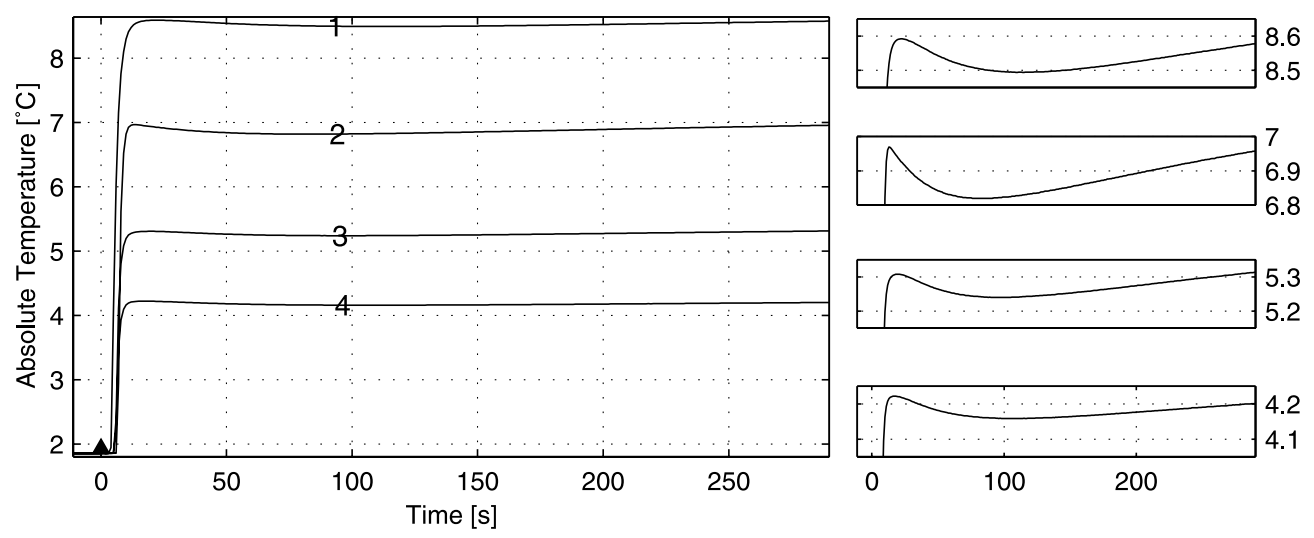

Fig. 9. Left: temperature records from four loggers mounted on the piston core barrel PC39. Right: enlarged temperature records which show the disturbed decay curve. The temperature scale for each plot is $0.2^{\circ} \mathrm{C}$.

4.73 and $6.81 \mathrm{~m}$ ) and show a feature which seems to be typical for gradient measurements on piston corers.

In the left side of Fig. 9 one sees a constant bottom water temperature followed by a high temperature increase and only a slight decay. This means that the sediment is rather warm so that the amount of frictional heating roughly equals the in situ sediment temperatures. It is striking that after 100-200 s, a temperature increase is superimposed on the decay curves. This can be seen more clearly from the enlargements on the right side of Fig. 9 plotted with a uniform temperature scale of $0.2^{\circ} \mathrm{C}$. The mean temperature increase is $0.1 \mathrm{~K}$ but has different amplitudes for each logger and is highest for logger no. 2 with $0.15 \mathrm{~K}$. Several causes may be responsible for the observed temperature rise:

- The maximum penetration velocity of a piston corer, as derived from acceleration monitoring during penetration, is eight times higher than the typical $1-\mathrm{m} / \mathrm{s}$ penetration velocity of a gravity corer (Villinger et al., 1999). The friction that occurs on the parts of the core barrel and attachment is proportional to the square of the velocity. Therefore, the amount of heating will be much higher and might be detected by the sensors. Moreover, the piston core barrel has a bigger diameter than the gravity core barrel which leads to a larger surface where friction occurs. This effect probably enlarges the heating as well.

- Another effect that probably has to be taken into account is the deformation of the sediment around the core barrel. A deep penetration causes severe disturbances of the sediments in the form of distortions and strains and changes in pore water pressures (Bahligh, 1986). The deformations are proportional to the penetration radius, the strain rates also depend on the penetration velocity. This deformation in the sediment might cause an internal heating when the deformation energy is converted into heat. By this effect, heat could be developed in a rather large area around the core barrel and closer to the sensor tip.

To locate the source of the temperature disturbance, the distance that a heat wave can travel during $100 \mathrm{~s}$ is estimated. It strongly depends on the thermal diffusivity of the material in question. In sediments with a thermal diffusivity in the order of $2 \cdot 10^{-7} \mathrm{~m}^{2} / \mathrm{s}$, the disturbance can cover around $5-10 \mathrm{~mm}$ within $100 \mathrm{~s}$. In steel with 100 times higher diffusivity, the distance amounts to 10 times that in sediment, that means up to 5$10 \mathrm{~cm}$. One possibility is that heating occurs on the fins and propagates from the attachment caps to the sensor tip through the sediment. The temperature rise can be small enough not to be detected in the case of the gravity corer but high enough in the case of the piston corer. Another possibility is that the heating stems from the core barrel itself or parts of the attachment and propagated through the steel and reaches the sensor tip that way. Numerical modeling of the complete system might help to clarify the phenomena observed. 

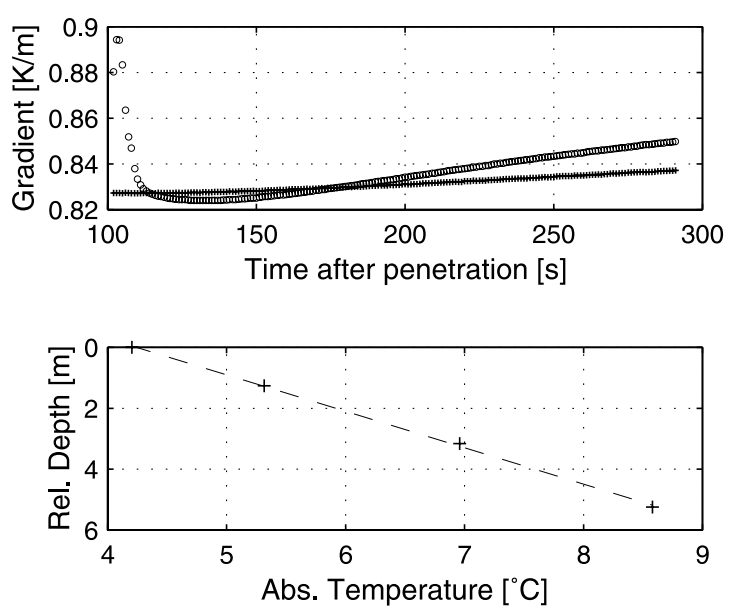

Fig. 10. Upper: gradient from piston core 39 obtained by extrapolation (o) and time-dependent gradient $(*)$. Lower: sediment temperatures taken from the last readings after $290 \mathrm{~s}$.

Despite these temperature disturbances, temperature gradients could be derived with the extrapolation and direct method. In the upper part of Fig. 10 the gradients calculated with these different methods are shown as a function of recording time whereas in the lower graph the sediment temperatures obtained by the direct method are illustrated. In this case, with the temperature disturbance beginning at $100 \mathrm{~s}$, the results from the methods show clear differences: as discussed in 3.2. Pogo style measurements with a 6-m lance, the extrapolation method reacts stronger to the temperature disturbances than the direct method. Up to $130 \mathrm{~s}$, the cooling trend dominates the extrapolation so that the calculated gradient falls below the 'direct' gradient. After $130 \mathrm{~s}$, the increasing temperature leads to an even stronger increasing gradient by the extrapolation. The direct method is more stable and varies only $2 \%$ with time and yields a gradient of $0.838 \mathrm{~K} / \mathrm{m}$ at the record's end which is shown in the lower graph. Although the direct method seems to be more reliable, it should be noted that both gradients agree within $2 \%$ in a time window from 120 to $280 \mathrm{~s}$.

For this data example, we also want to demonstrate the determination of thermal conductivity and the calculation of heat flow in order to compare the results with a conventional heat flow de-
Thermal conductivity $(\mathrm{W} / \mathrm{mK})$

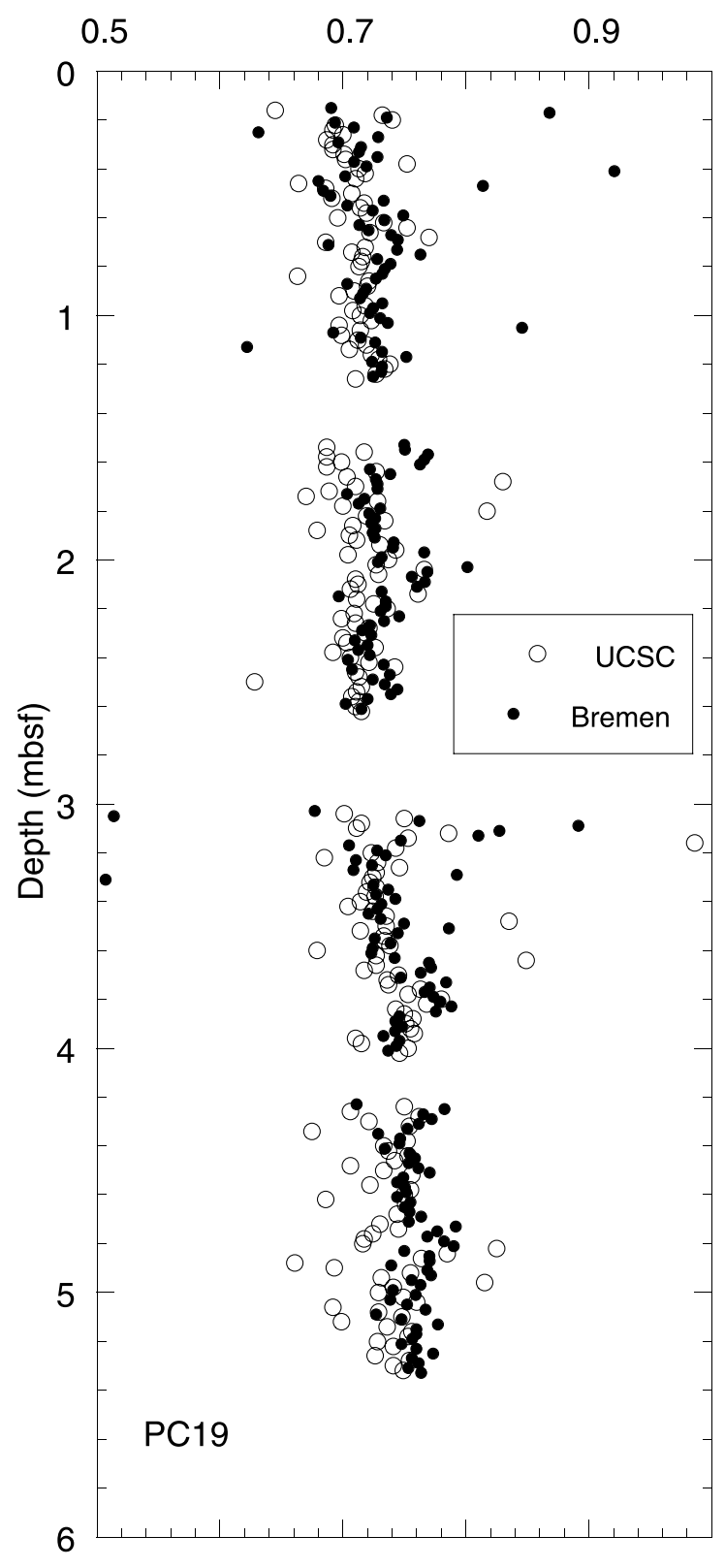

Fig. 11. Thermal conductivities measured with the UCSC and Bremen instruments.

termination. Core material from a nearby coring location has been used to determine the physical properties and thermal conductivity of the sediment. Piston core 19 was located $800 \mathrm{~m}$ away from PC39 which was described here. The thermal 
conductivity has been determined by needle probe measurements in the laboratory with both the pulsed (Lister, 1979) and the continuous heating method (Von Herzen and Maxell, 1959). The measurements were performed on the full core in distances of $1 \mathrm{~cm}$. In Fig. 11, the data from the UCSC (University of California, Santa Cruz, CA, USA) instrument and Bremen instrument are combined. The two data sets agree well, with minor exceptions in all sections. The mean thermal conductivity is about $0.72 \mathrm{~W} / \mathrm{mK}$ and slightly increasing with depth. Gaps in the data are core section borders where measurements are influenced by the end caps and therefore have only been performed in a distance of about $5 \mathrm{~cm}$ from the end caps of each section. The high values near $3 \mathrm{~m}$ below seafloor correspond to an ash layer found after cutting the core in halves. Other single high values could not be linked to structural features, but probably can be correlated in the future when the additional density and porosity samples have been processed. With a mean thermal conductivity of $0.72 \mathrm{~W} / \mathrm{mK}$ assumed for all depths, the heat flow at station PC 39 is $603 \mathrm{~mW} /$ $\mathrm{m}^{2}$. Both the conductivity and the calculated heat flow could be reproduced by a nearby conventional heat flow determination.

During the cruise EW0104 we successfully deployed six piston corers equipped with three to six outrigger probes with a minimum loss of instruments. The fin-like attachments withstood the penetration forces without slipping along the core barrel except from one station where the core hit an ash layer, the lowermost three fins slid along the core barrel and one of them was lost. Nevertheless, data were recorded but could not be processed because of the unknown position during penetration. In another deployment, the sensor tip of deepest sensor was strongly bend but still recorded correct data. On all other locations, the loggers delivered reliable sediment temperature gradients up to a depth of $6.8 \mathrm{~m}$ below the weight stand.

The high penetration velocity has two effects: on the one hand it might cause more damage to the loggers, so that more attention has to be paid on the mechanical robustness and firm attachment of the fins, and on the other hand it might cause secondary thermal disturbances. Since this unwelcome temperature increase does not influence the measurements too much, data can still be processed and gradients derived.

\section{Results and outlook}

The MTL is an easy to handle and reliable instrument for high precision temperature measurements in a deep sea environment. The instruments are robust, small and easy to prepare for deployment. Programming the instruments and downloading data did not cause any problems, and recording never failed by the rough handling as long as the battery voltage was high enough.

The data obtained from the loggers are of a high quality: no scatter can be detected on all records and even with a loss of battery power the results are reliable up to the abrupt end of the record. The absolute accuracy was increased to several $\mathrm{mK}$ with the help of the calibration in a water bath. The variable sample rate allows different logging durations; we mostly used 1-s sample interval in order to achieve a high resolution in time. The small thermal time constant of $2 \mathrm{~s}$ enables to detect even small and short temperature changes.

We used the MTLs on four cruises from 1999 to 2001 to investigate temperature gradients in deep sea sediments. The instruments have been deployed 28 times on a lance as well as 10 times on a gravity and six times on a piston core. All deployments on the lance were successful, and only on one piston core some instruments have seriously been damaged. Reliable data have been recorded on all other instruments.

In all cases presented in this paper we assumed a vertical penetration of the lance or core barrel, because mostly tilts below $5^{\circ}$ have been measured which are negligible for a tilt angle correction.

To derive reliable gradients, it is important to measure temperatures over at least 3 min, ideally 5 min after penetration and not to take into account records less than $150 \mathrm{~s}$ after the penetration. The gradients can be determined with the direct method without any processing or with the extrapolation method. The deviations between 
the results are in most cases $\pm 2 \%$ or less and since the direct method is the less sensitive but more stable method, it can be used to yield reliable results.

As far as measurements with the conventional heat flow probe have been performed, the calculated gradients were comparable within $\pm 3 \%$, taken into account the different locations of these measurements. On cruise SO146, loggers were mounted on the strength member of the conventional heat probe and delivered the same gradient within $\pm 8 \%$ (Kaul, 2001, personal communication). It has to be emphasized that this result was obtained during experimentally changing the design of the attachments and may be influenced by an unfortunate choice of geometry. Nevertheless, the deviation is still in the order of the reproducibility of conventional heat flow determinations (Kaul, 2001, personal communication).

With the help of needle probe measurements performed on each core on the cruises SO145 and EW0104, the thermal conductivity could be determined on recovered core material and the heat flow calculated.

Our very positive experiences encourage us to use the MTLs as standard equipment on subsequent cruises and to make further effort to derive gradients over an even larger depth scale. Other possible deployments for the MTLs are for example the long term monitoring in the deep sea or investigation in bore holes. The possibility of linking temperature to visual information is given by fixing the logger on the pilot weight of a camera sledge. This method provides precise temperature measurements with a high spatial resolution in the bottom water very close to the seafloor and has also been successfully tested on cruise SO146.

\section{Acknowledgements}

Initial development of the loggers was possible by a generous grant from MARUM (University of Bremen, Germany). The authors would like to thank the captains, officers and crews of R/V 'Sonne' during the cruises SO145, SO146 and SO149 who provided excellent ship handling and support during the heat flow stations. We especially thank Norbert Kaul and Bernd Heesemann for providing data from SO149 and helping on all the other cruises. We also acknowledge the effective collaboration with the 'TicoFlux' group, namely A. Fisher, R. Harris, C. Stein, K. Wang, J. Wheat, A. Cherkaoui, M. Hutnak and R. Cleary. We thank this group for the invitation to take part in their cruise and the excellent support. The authors would like to thank captain and crew of R/V 'Maurice Ewing' as well for their help and support. Participation for M.P. was made possible by a Grant of the Deutsche Forschungsgemeinschaft (Vi 133/5-1). The research cruises on board R/V 'Sonne' were funded by the German Ministry of Science and Technology: SO145 with Grant 03G0145A, SO146 with 03G0146C and SO149 with 03G0149A.

\section{References}

Bahligh, M.M., 1986. Undrained deep penetration, I: shear stresses. Geotechnique 36, 471-485.

Bennett, A.S., 1972. The calibration of thermistors over the temperature range $0-30^{\circ} \mathrm{C}$. Deep-Sea Res. $19,157$.

Bialas, J., Kukowski, N., Scientific Party SO146, 2000. Geomar Report No. 96, Cruise Report SO146-1 and -2, GEOPECO, Geophysical Experiments at the Peruvian Continental margin, investigations of tectonics, mechanics, gashydrates, and fluid transport, Arica-Talcahuano, March 1-May 4, 2000. Geomar Research Center for Marine Geosciences, Christian Albrechts University, Kiel.

Blackwell, J.H., 1954. A transient flow method for determination of thermal constants of insulating materials in bulk. J. Appl. Phys. 25, 137.

Fisher, A.T., Iturrino, G., Jolly, G., Dolling, R., Daniel, D.N., Stein, J., Jin, W., 1996. New autonomous outrigger probes used for the collection of thermal and chemical gradient data at the same location. EOS 77 (Suppl.), 256-257.

Fisher, A., Silver, E., Wheat, G., Harris, R., Stein, C., Wang, K., Underwood, M., Moser, C., Hutnak, M., Cherkaoui, A., Kelly, R., Pfender, M., Friedmann, P., Bodzin, R., Cleary, R., Stewart, Y., Jones, K., 2001. Preliminary Cruise Report, TicoFlux I Expedition, R/V Maurice Ewing 0104, 14 April19 May 2001.

Fisher, A., Harris, R., Stein, C., Wang, K., Hutnak, M., Cherkaoui, A., Pfender, M., Cleary, R., Silver, E., Wheat, G., Bodzin, R., Underwood, M., Moser, C., Kelly, R., Friedmann, P., Stewart, Y., Jones, K., 2001. Heat flow on the incoming plate offshore Nicoya, Costa Rica margin: Implications for hydrothermal circulation and the thermal state of 
the subducting plate. EOS Trans. Am. Geophys. Union 82(47), Fall Meet. Suppl., Abstr. T22C-0922.

Gerard, R., Langseth, M.G. Jr., Ewing, M., 1962. Thermal gradient measurements in the water and bottom sediments on the Western Atlantic. J. Geophys. Res. 67, 785-803.

Hyndman, R.D., Davis, E.E., Wright, J.A., 1979. The measurement of marine geothermal heat flow by a multipenetration probe with digital acoustic telemetry and in situ thermal conductivity. Mar. Geophys. Res. 4, 181-205.

Kaul, N., Rosenberger, A., Villinger, H., 2000. Comparison of measured and BSR-derived heat flow values, Makran accretionary prism, Pakistan. Mar. Geol. 164, 37-51.

Langseth, M.G. Jr., 1965. Techniques for measuring heat flow through the ocean floor. In: Lee, W.H.K. (Ed.), Terrestrial Heat Flow. American Geophysical Union, Geophys. Monogr. Ser. 8, 245-271.

Lister, C.R.B., 1979. The pulse-probe method of conductivity measurement. Geophys. J. R. Astron. Soc. 57, 451-461.

Lister, C.R.B., Sclater, J.G., Davis, E.E., Villinger, H., Nagihara, S., 1990. Heat flow maintained in ocean basins of great age: investigations in the north-equatorial West Pacific. Geophys. J. Int. 102, 603-630.

Spiess, V., Villinger, H., Zühlsdorff, L., Arnold, W., Bartels, T., Böke, W., Heesemann, B., von Larcher, T., Matschkowski, T., Meffert, T., Müller, H., Oetjen, H., Pfender, M., Rohr, K., Schwenk, T., 2001. No. 179, Report and preliminary results of R/V Sonne cruise SO149, Victoria-Victoria, 16-8-16-9-2000. Fachbereich Geowissenschaften, Universität Bremen, Bremen.

Steinhart, I.S., Hart, S.R., 1968. Calibration curves for thermistors. Deep-Sea Res. 15, 497.

Villinger, H., Grigel, J., Heesemann, B., 1999. Accelerationmonitored coring revisited. Geo-Mar. Lett. 19, 275-281.

Villinger, H., Scientific Party SO145, 2000. No. 154, Report and preliminary results of Sonne-cruise 145-1, Balboa-Talcahuano, 21-12-1999-28-01-2000. Fachbereich Geowissenschaften, Universität Bremen, Bremen.

Von Herzen, R.P., Maxell, A.E., 1959. The measurement of thermal conductivity of deep-sea sediments by a needle probe method. J. Geophys. Res. 64, 1557-1563. 